\title{
The criteria for capping agents selection for DNA-encoded library technologies (DELT) needs
}

\section{R. Smaliy, A. Pashenko, D. Volochnyuk, S. Ryabukhin}

\section{Introduction and Aim}

DELT stands remarkable position in early drug discovery toolkit as rapid and inexpensive approach to generating large collections of combinatorial compounds. It is notable, that the size of the libraries created using DELT sometimes outnumber the size of the common combinatorial HTS libraries by several orders of magnitude. ${ }^{1}$ As a consequence of enlarged size, the design of DELs demands significant numbers of eligible mono-functional building blocks (known as modifiers, or capping agents) available. Despite the prevalent belief that most of the HTS building blocks could be used for DELT, there are selection benchmarks that edge the number of actually DELT-compatible blocks. Reactivity in aqueous, non-acidic conditions below $90^{\circ} \mathrm{C}$ with high conversion and selectivity is the hallmark for the DEL-compatible capping molecules. ${ }^{2}$

Herein we report the chemoinformatical selection algorithm, that allows pre-validating the capping BBs. The general criteria account molecular weight, substituents compatibility, steric hindrance and chirality. The custom filters enable selection basing on lipophilicity, number of rotating bonds or basicity. The statistical analysis of fraction of the "likely DELT compatible" capping molecules comparing to the overall array of stock building blocks in the eMolecules ${ }^{3}$, Chemspace and Enamine datasets is given.

Typical DEL sequencing for 4-step cycle

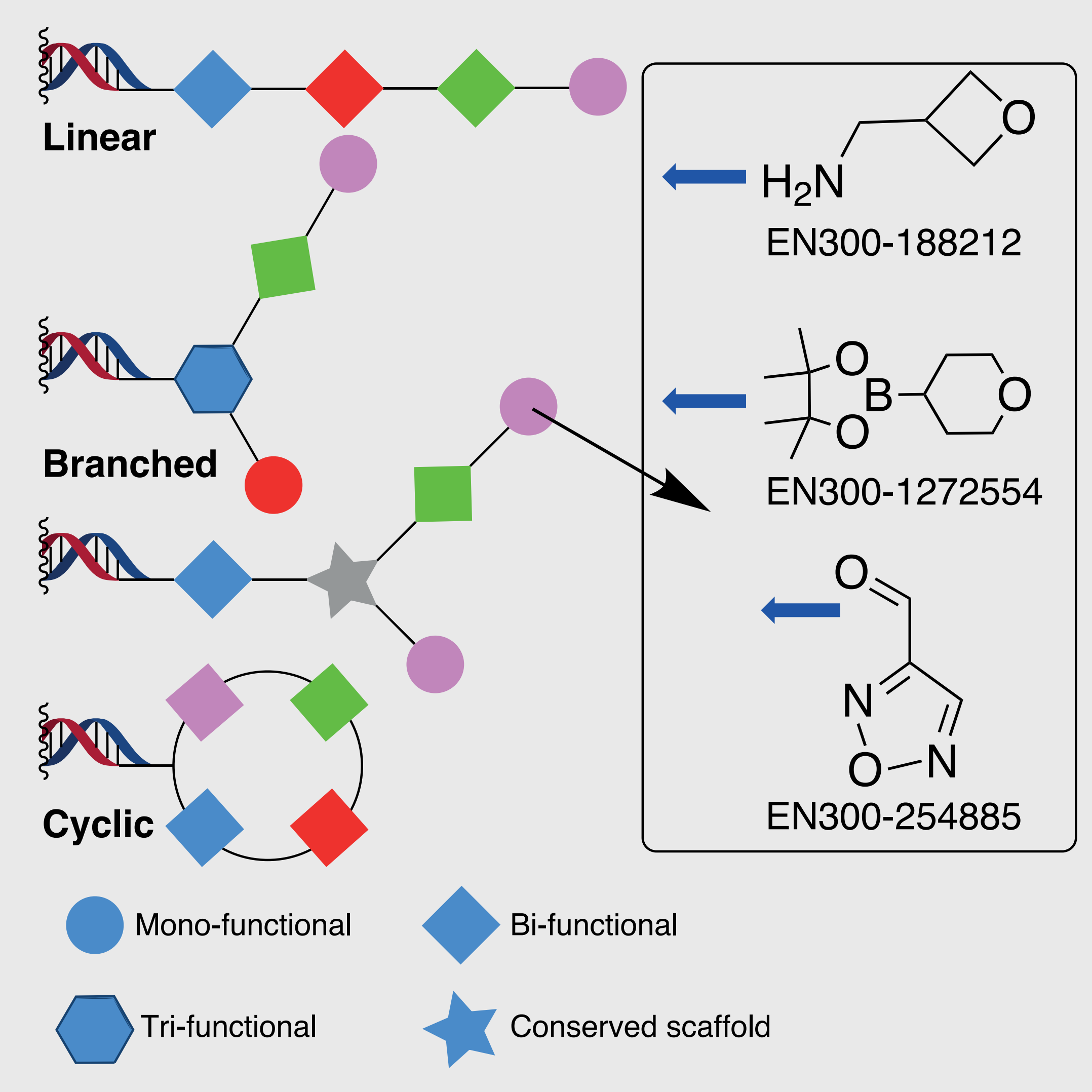

\section{Statistical analysis of DEL-compatible mono-functional BBs availability}

DEL-compatible capping agents availability

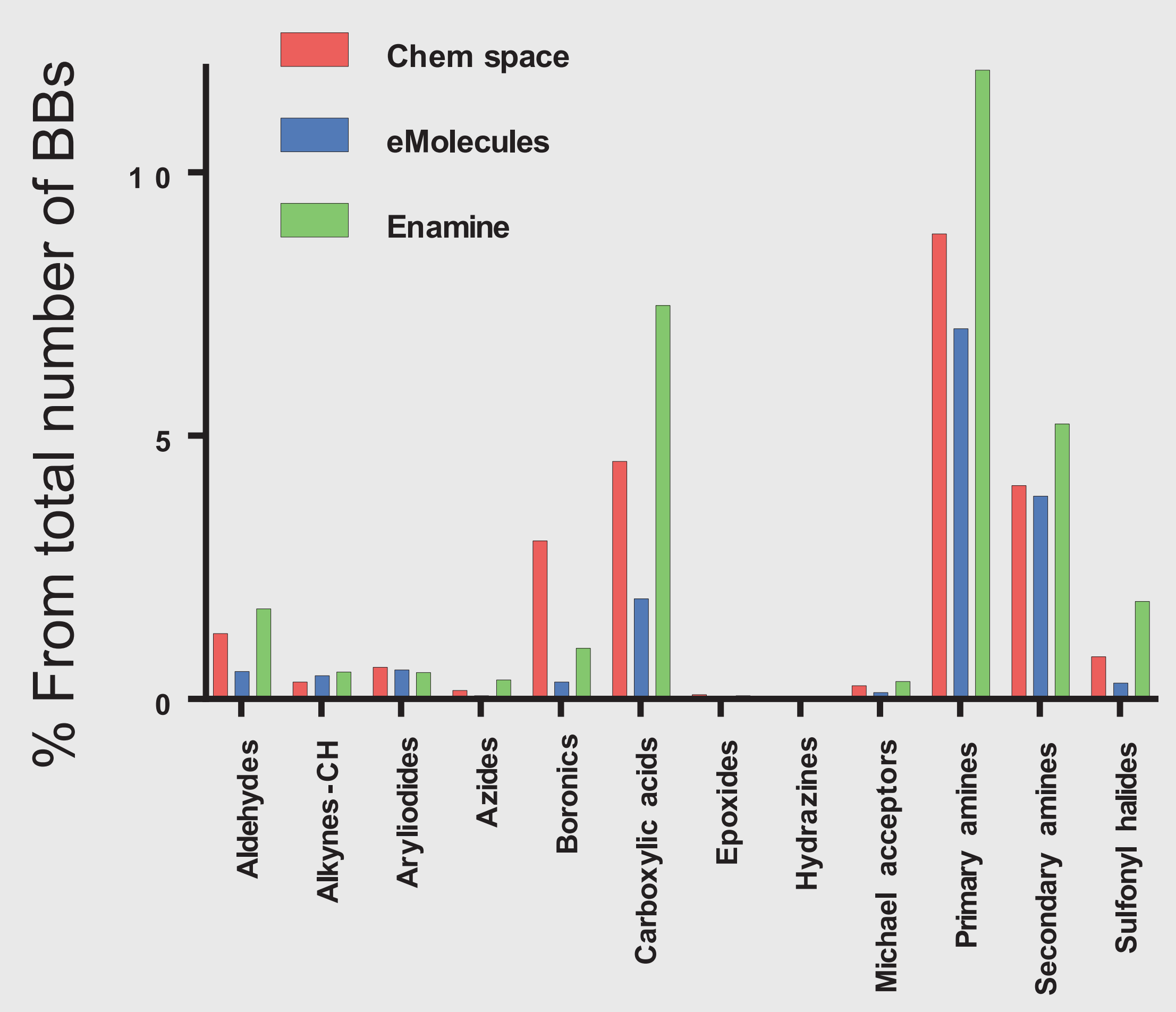

Database analysis for widespread class of capping agents and one of the scarcer
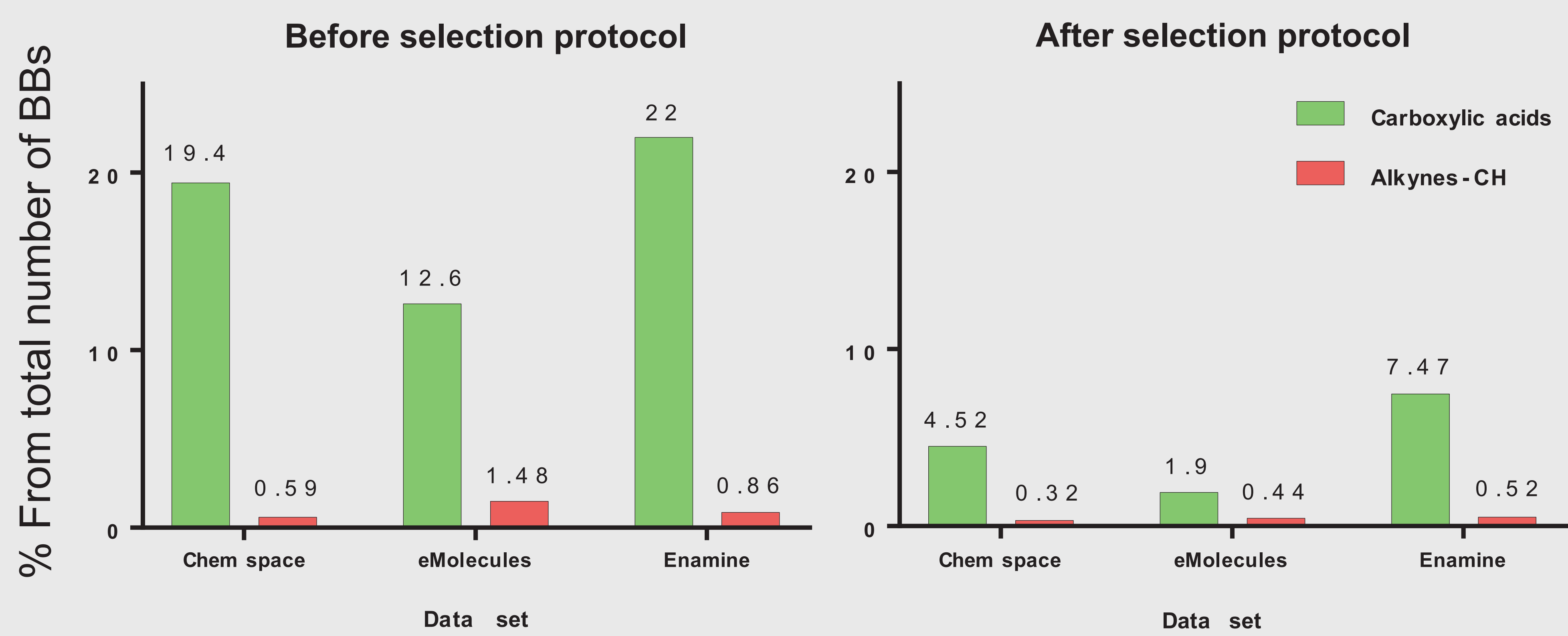

\begin{tabular}{|l|r|r|r|}
\hline & Chemspace & eMolecules & Enamine \\
\hline Total BBs count & 468,061 & $6,908,533$ & 156,927 \\
\hline Carboxylic acids (unsorted) & 90,908 & 871,856 & 34,511 \\
\hline Alkynes-CH (unsorted) & 2,748 & 102,296 & 1,348 \\
\hline
\end{tabular}

\section{DEL-compatibility criteria: general overview on selection protocols for capping BBs}

\section{General and custom selection criteria with examples}

General exclusion criteria:

- Interfering functional groups

- Racemic compounds or compounds with unidentified chirality

- Functional groups with reactivity significantly decreased within the given class (for instance deactivated by neighboring EWG)

- Molecular weight above $125 \mathrm{Da}$ (when targeting 500 and less da screening compounds $)^{4}$

- Steric hindrance

Custom exclusion criteria

- Fraction of saturated carbons $\left(F s p^{3}\right)$

- Number of rotation bonds below 4

- Lipophilicity

- Basicity

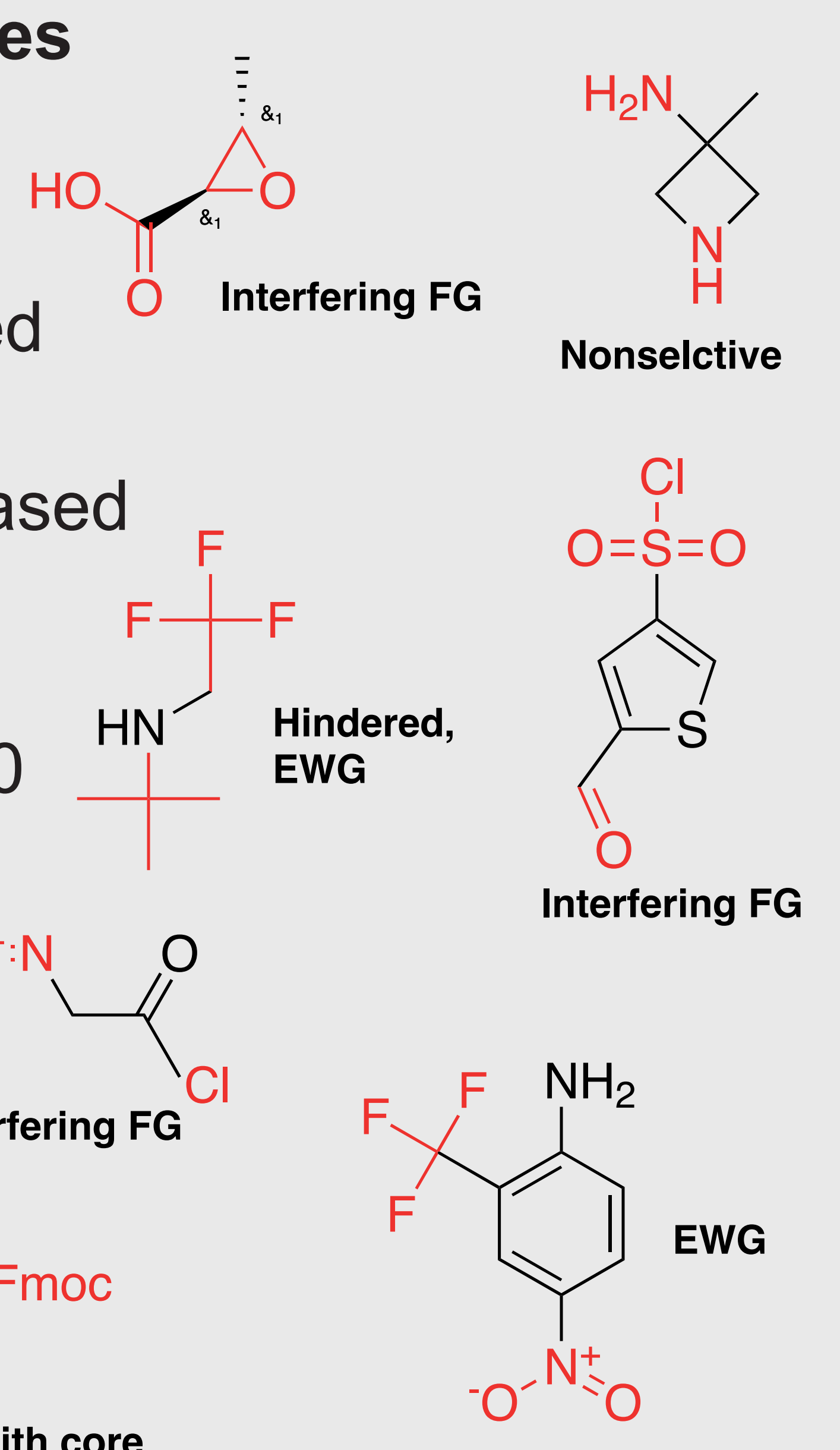

PG nonortogonal with core

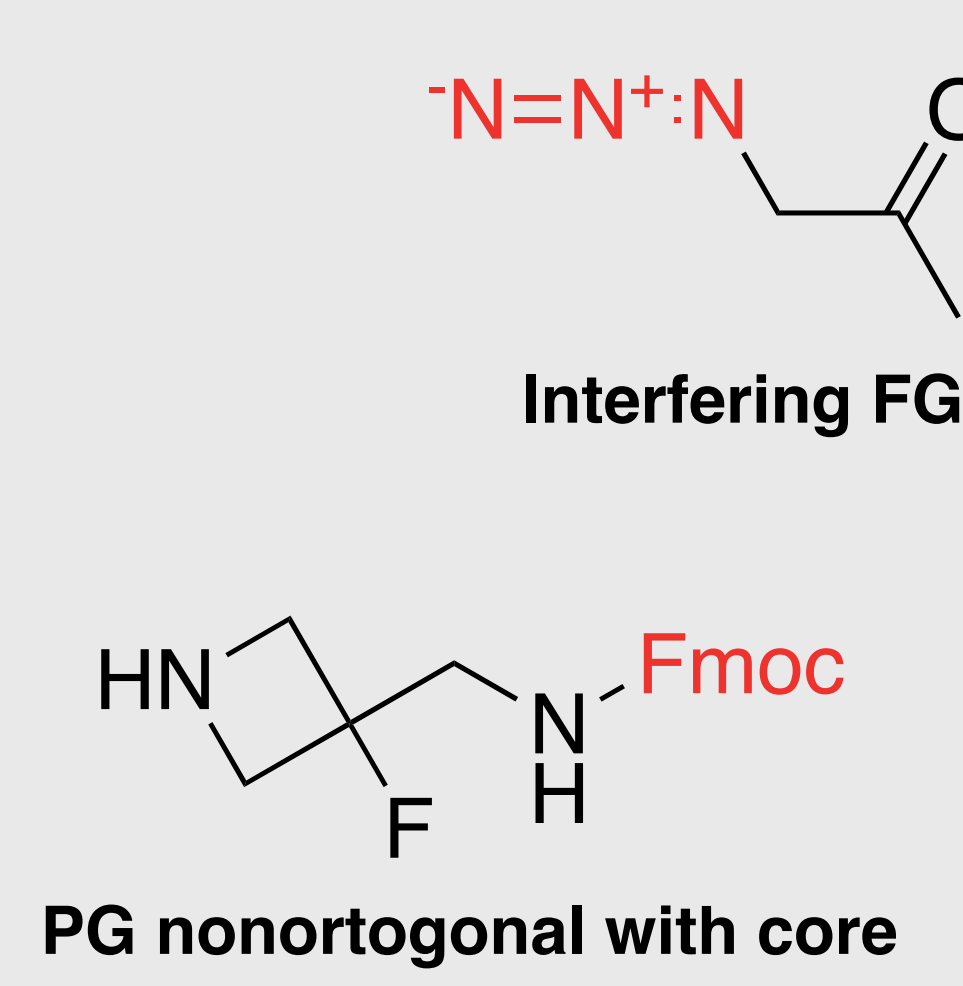

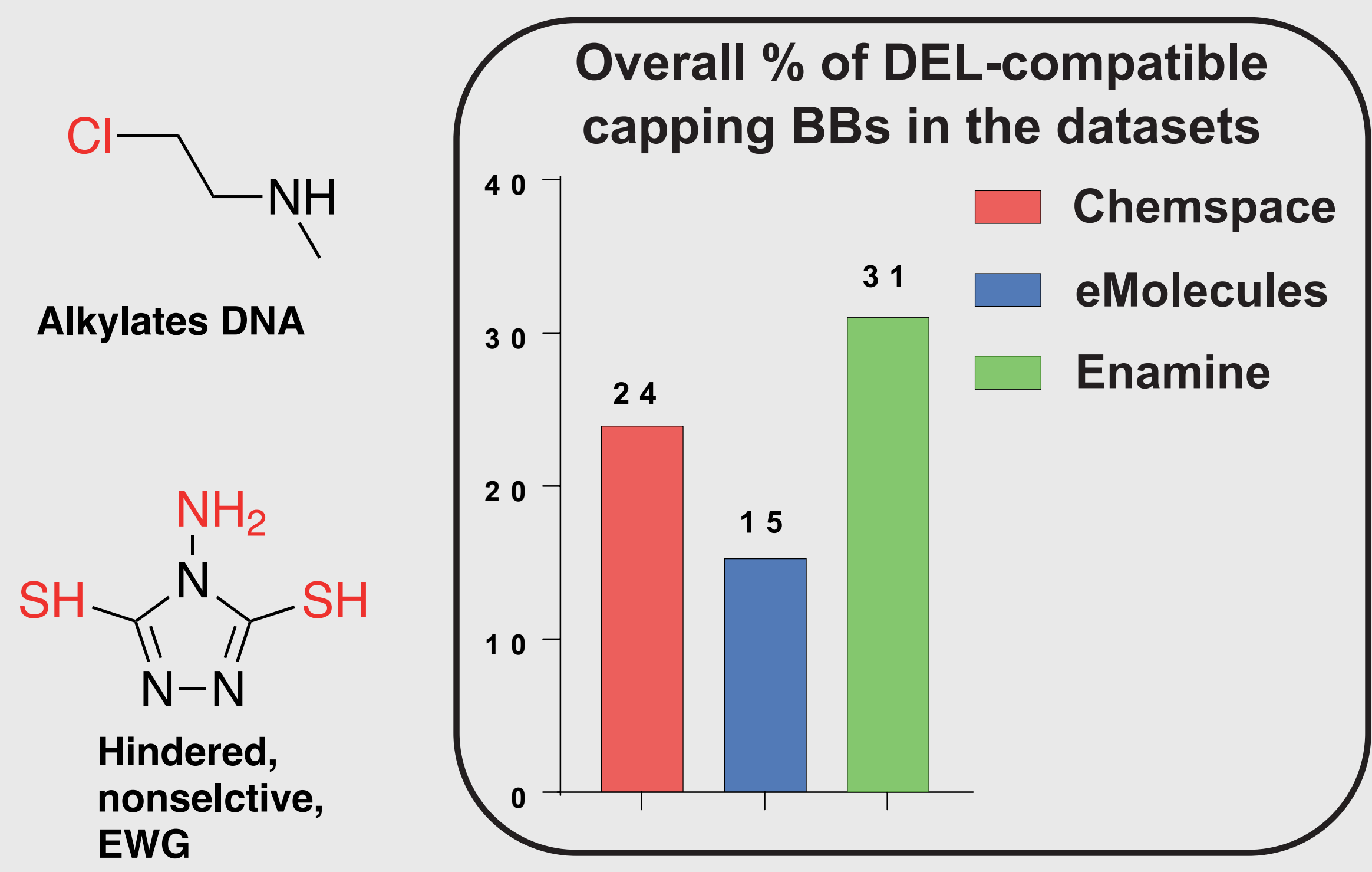

EWG
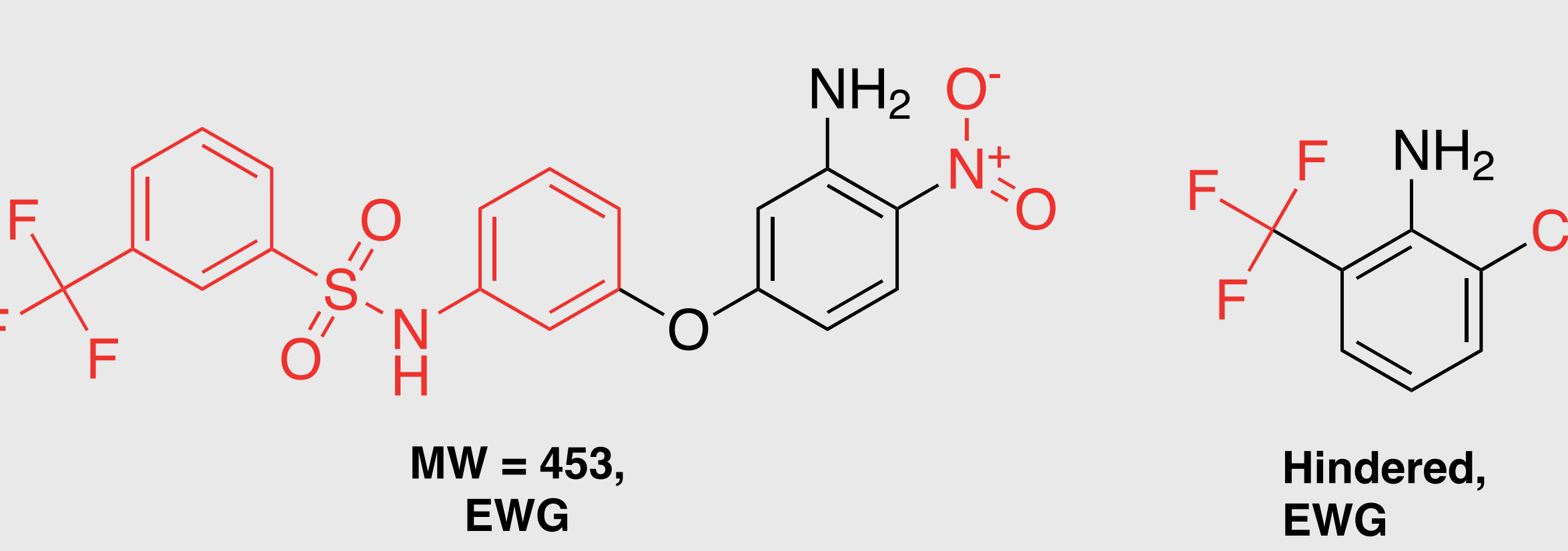

\section{Contact}

